\title{
CHESHIRE CHAT: KEEPING UP WITH THE TIMES
}

\author{
Peter Munday ${ }^{1}$ \\ ${ }^{1}$ Cheshire CCG
}

\section{CCGS AND THE PUBLIC:}

Clinical Commissioning Groups (CCGs) have a legal obligation to engage with and to consult their resident populations. This can be challenging and expensive across a wide geographical area, even at the best of times. The "lockdown" and the subsequent social distancing requirements of the COVID-19 pandemic have inevitably presented further barriers to organising events.

It is important to provide patients, public and stakeholders with a voice, in order to shape and drive local healthcare. However, public interest can be difficult to stimulate and gauge, requiring residents with already busy lives to sacrifice their time in the day or evening. Wherever physical travel is required, inclusiveness and accessibility are always key concerns.

The newly merged NHS Cheshire CCG has recently adopted an inexpensive remote-conferencing option. This has allowed some events to continue as usual, and has also provided a platform to improve the extent of public participation and quality of events. This decision was partially motivated by cost; a room-hire budget for even a relatively modest in-person event can easily run to $£ 500$, particularly if sophisticated projection and amplification equipment is required. Moreover, staff time and expenses carry a much higher cost, for they must not only travel to and host the event, but also document, process and analyse what is said during and afterwards. Nevertheless, the CCG are aware that online events are not suitable for everybody and are therefore working with local authorities to develop Digital Inclusions plans. These intend to explore ways to reach out to the community and support those who are not 'digitally connected' until it's possible to resume meeting face to face.

\section{CCGS AND COVID-19:}

The COVID-19 pandemic has entailed rapid change in local healthcare systems, necessitating equally swift engagement and communication with the public. This is likely to continue into the future; CCGs must learn to adapt quickly to the new environment.

As was typical of other industries, the NHS Cheshire CCG moved very quickly to tele-conferencing options for internal meetings. "Cheshire Chat" was established after a review of different digital engagement channels. It is an online webinar event using the GoTo Webinar application, which the CCG had already purchased for Governing Body meetings. This allowed live broadcast of the event, desk top presentation 
and also questions and feedback from the audience. Multiple presenters were co-ordinated by designated "organisers", allowing the proceedings to be conducted entirely virtually.

The software can provide useful analytics and allows the circulation of post-meeting surveys to supplement the synchronous material. Moreover, the application is accessed online, therefore no proprietary software is required by any member of the public wishing to take part. Most importantly, the process is self-documenting as the live event and written comments may be recorded if required. Both the full version or excerpts of recordings may be made available for viewing or listening after the event.

The first event dealing with topics on Mental Health, Cancer Services and Primary Care had 60 members of the public registered - more than twice the usual number of attendees than an in-person event typically attracts. Promotion was through:

\section{- Social media}

- Email invites to stakeholders (although the event was open to anyone)

- Copies sent to local Healthwatch for their website and social media

- Tiktok videos of commissioning colleagues to share on all channels

- A link to a survey where people who could not attend, could still leave comments and questions.

The "topical" approach also allows the public to focus on only those areas which interest them. The Webinar format also supports a greater number- and therefore a more inclusive pool- of panel members in a far more cost-effective way.

\section{THE FUTURE OF CHESHIRE CHAT:}

The feedback received so far has been excellent. Typical comments have been:

"A great way to feel connected, to hear the latest updates and to have an opportunity to ask questions."

"Thanks for hosting this on-line \#CheshireChat"

"Good to hear how CCG are seeing the benefits of some virtual/technological delivery"

Following the undoubted success of the format, further events will be hosted every eight weeks. The same structure will be followed, with three discussion topics across a onehour time slot. This will result in online events being hosted at around twice the frequency of in-person events in previous years. The intention is to continually receive the views, comments and feedback of local attendees, which will in turn shape the future commissioning intentions of the CCG. This arrangement will also be used to supplement formal public consultation in the future if necessary.

While there are undoubted savings in costs, the biggest gain in "value" is from the robustness and quality of the process, and the chance to involve a greater proportion of the population. 